\title{
Chicken Swarm Optimization based Optimal Channel Allocation in Massive MIMO
}

S Nisharani ( $\nabla$ nisharani.84@gmail.com )

Kamaraj College of Engineering and Technology

G Indumathi

Mepco Schlenk Engineering College

\section{Research Article}

Keywords: Chicken Swarm Optimization (CSO), Energy Efficiency (EE), Massive Multiple-Input MultipleOutput (MIMO), Resource Efficiency (RE) Metric, Spectral Efficiency (SE)

Posted Date: July 13th, 2021

DOI: https://doi.org/10.21203/rs.3.rs-632638/v1

License: (1) This work is licensed under a Creative Commons Attribution 4.0 International License. Read Full License 


\title{
Chicken Swarm Optimization based Optimal Channel Allocation in Massive MIMO
}

\author{
${ }^{[1]}$ Nisharani S, ${ }^{[2]}$ Dr.Indumathi G \\ ${ }^{[1]}$ Assistant Professor, Department of ECE, Kamaraj College of Engineering and Technology, \\ Tamilnadu, India, nisharani.84@gmail.com \\ ${ }^{[2]}$ Professor, Department of ECE, Mepco Schlenk Engineering College, Tamilnadu, India, \\ gindu@mepcoeng.ac.in
}

\begin{abstract}
Energy Efficiency (EE) plays a significant role in the progress towards the Fifth-Generation (5G) wireless communication networks. Due to the higher Spectral Efficiency (SE) and EE, Massive Multiple-Input Multiple-Output (MIMO) is a promising model for the 5G networks. In this work, a Channel Selection (CS) scheme is proposed by selecting the optimal channel using the Chicken Swarm Optimization (CSO) algorithm. A massive MIMO model is implemented by considering the SE, EE and Resource Efficiency (RE). The main objective is to optimize the beam-forming vectors and power allocation for all the users. The RE metric considering the multi-objective function can be defined to develop an effective and robust design with balanced SE and EE. The objective function for generating the optimal beam forming vectors is satisfying the Signal to Interference-Plus-Noise Ratio (SINR) constraints. The CSO Algorithm is applied to generate the beam-forming vectors and power allocation, based on the channel characteristics. The channel state information is predicted and a projection matrix with channel estimation framework is formed. The selection of the index sets in the iteration process provides the optimized channel. Data transmission is performed through the optimal channel. From the comparative analysis, it is observed that the proposed CS scheme provides better SE and EE than the existing CS schemes.
\end{abstract}

Keywords: Chicken Swarm Optimization (CSO), Energy Efficiency (EE), Massive MultipleInput Multiple-Output (MIMO), Resource Efficiency (RE) Metric, Spectral Efficiency (SE)

\section{Introduction}

In the former decade, there has been a considerable focus on improving the SE and EE of the wireless communication system based on the increasing demand for the multimedia applications. Owing to the high SE [1] and EE [2,3], massive MIMO is the perfect model for the $5 \mathrm{G}$ networks. The main idea of the Massive MIMO is equipping the Base Stations (BSs) with multiple antenna elements at the transmitter and receiver and grouping them together to provide better throughput and SE. It also provides new spatial degrees of freedom for serving multiple user terminals simultaneously on the same time-frequency channel. With the increase in the number of antennas, the communication performance in terms of data rate and link reliability gets improved simultaneously [4-7].

Though there is a significant improvement in the SE with a large number of antennas supporting huge number of users linked in the same radio channel. This results in the drastic increase in the power consumption and decrease in the EE of the MIMO systems [8]. Also, the operational cost and power consumption increases with the increase in the large number of antennas equipped with Analog to Digital Converters (ADCs) and Digital to Analog Converters (DACs), deployed at the BS $[5,7,9]$. With the requirement of huge bandwidth and high sampling rates in the next generation wireless communication systems, high-speed 
ADCs are unavailable or too expensive for the practical usage [10]. Usage of the lowresolution ADC/DACs is suggested for the massive MIMO systems [11-14]. One-bit ADC/DACs requires minimum hardware cost and power consumption, due to a single comparator, no need for the linear amplifiers and automatic gain control. Existing systems have conducted experimental verification about the benefits of implementing the lowresolution/mixed ADCs to reduce the power consumption [15-17].

According to the study conducted by Ngo et al. [3], the EE value is related to the transmit power of the users. However, in the practical massive MIMO systems, the energy consumption should involve both the power consumed in the radio frequency circuit and the radiated power. Chen et al. [18] measured the tradeoff between the SE and EE, to maximize the EE value by assigning the time and power resources. The proper beam-forming design helped in improving the EE value but resulted in the reduced SE value [19]. It is concluded that the rising of the EE value caused decrease in the SE value. Hence, a novel technique to maintain the tradeoff between the EE and SE in massive MIMO system is required.

The swarm intelligence based optimization and evolutionary algorithms are found to be effective for the optimization of the beam patterns. Previously, Genetic Algorithm (GA)[20], Particle Swarm Optimization (PSO)[21], Ant Lion Optimization (ALO)[22], Flower Pollination Algorithm (FPA)[23], hybrid Invasive Weed Optimization (IWO)/Wind Driven Optimization (WDO)[24], PSO-Gravitational Search Algorithm-Explore (PSOGSA-E) [25] and Firefly Algorithms (FA) algorithms [26]were applied for the beam pattern optimization. Due to the slow convergence rate and less stability of the existing swarm intelligence based methods, improved CSO algorithm is applied to find the optimal channel. CSO inherits the advantages of the PSO and Differential Evolution (DE) algorithms. Owing to the diversified movements of the chickens between multiple groups, there is a highly efficient exploration of the search space and obtain a great balance between the randomness and determination for finding the optima. For a specific hierarchical order, the entire swarm might coordinate together like a team to search food. This could be associated with the optimization of the objective problems through the efficient extraction of the swarm intelligence of the chickens. Thus, the performance of the CSO algorithm is high.The CSO algorithm is applied for the optimal channel allocation in the massive MIMO system.In the proposed work, the parameters and population are initialized and the fitness function is estimated. The optimal solutions are obtained and the positions of the hens, roosters and chickens are updated. The global optimal positions are obtained and then it is checked whether the maximum number of iterations are reached. If the maximum number of iterations is reached, the optimal channel output is obtained. Else, the process is returned to the estimation of the fitness function.Through the optimization of the power allocation and beam forming vectors, the better tradeoff between the SE and EE in the massive MIMO system is achieved.

The major objectives of the proposed work are

1. To develop an improved massive MIMO model with a balanced trade-off between SE and EE.

2. To construct a trade-off between SE and EE by optimizing the power allocation and beam-forming vectors.

The structure of the paper is organized as follows: Section 2 provides a brief review of the existing research works related to the massive MIMO networks. Section 3 describes the proposed work including system model, objective model, optimal power allocation and 
beam-forming vectors and CS scheme with the CSO algorithm. Section 4 discusses the comparative analysis of the proposed CS scheme with the existing CS algorithm and CS with mixture of Gaussian cluster model, and section 5 concludes the work summarizing the key ideas.

\section{Literature Review}

Zhang et al. $[15,16]$ considered multiple pairs of amplify and forward two-way relay channel to exchange information through a full-duplex relay with huge number of antennas. Four power-scaling schemes were proposed based on the Maximum Ratio Combining (MRC)/MRT and ZF Reception (ZFR)/ZF Transmission (ZFT) at the relay. The asymptotic $\mathrm{SE}$ and $\mathrm{EE}$ for the proposed schemes were quantified, when the number of relay antennas reaches infinity. The loop interference could be reduced by decreasing the transmission power under the relay antennas. Also, the inter-pair and inter-user interferences were eliminated in the huge number of antennas. The proposed power-scaling schemes achieved good performance tradeoffs between the SE and EE.

Li et al. [27] investigated the channel estimation problem and determined the achievable uplink rates for the massive MIMO system in which one-bit ADCs are provided in the base station. A simple and perceptive expression for the Linear Minimum Mean-Square-Error (LMMSE) channel estimator was derived to obtain the closed-form expression for the lower bound of the achievable uplink rate. Experimental results proven that the proposed estimator outperformed the near maximum likelihood estimator.

He et al. [28] devised an easily adaptable Device-to-Device (D2D) power management strategy. A dynamic power control solution was introduced to efficiently avoid the interference between the cellular and D2D tier. An analytical approach is developed to evaluate the SE and EE in massive MIMO networks, by considering the maximum transmission power constraints at the cellular users and D2D transmitters. A tractable approach was developed to provide the exact expressions for computing the SE of the area of the cellular and D2D tier. Experimental outcomes confirmed that the performance of the proposed approach was efficient.

Patcharamaneepakorn et al. [29] studied about the spatial modulation (SM) schemes in the multi-cell multi-user massive MIMO systems. A suboptimal detection algorithm was proposed based on the linear processing technique. The probability of detecting antenna combinations was analyzed and used to approximate the sum-rate performance with the channel conditions. The basic trade-off between SE and EE was also investigated. Though with less SE rate, SM scheme with a single active antenna per user was shown to be the most effective energy efficient transmission mode.

Yang et al. [30] examined the SE and EE for a massive MIMO multiple-pair two-way amplify-and-forward relaying system. In this system, multiple pair of users exchange the information through a relay station with large scale antennas. It was assumed that the imperfect CSI was available and MRC/MRT was applied at the relay station. The asymptotic SE and EE were quantified under the common power scaling schemes applied to scale down the transmission power at each user and relay station. A closed-form SE expression was obtained approximately. The experimental results depicted that with the usage of massive relay antennas, the transmit power at each user and the relay station could be scaled down, with a non-vanishing SINR. 
Xin et al. [31] analyzed the performance of massive MIMO systems with the pilot contamination. A closed-form approximation of the Area SE (ASE) for uplink multi-cell multi-user massive MIMO systems was derived using a uniformly distributed user location model. The area EE (AEE) in a cell was obtained by considering a practical power consumption model. The ASE and AEE results were used to investigate the parameters of massive MIMO systems.

Tan et al. [32] derived an approximated upper bound on the achievable SE and EE of massive MIMO for the hybrid analog/digital pre-coding architectures based on the phase shifters. The BS has a perfect CSI. The baseband processing was performed based on the ZF pre-coding. It was found out that the number of BS antennas, users and Signal-to-Noise Ratio (SNR) were increased with the increase in the total achievable SE. An algorithm was proposed to generate the corresponding quantized matrix and hybrid architectures were studied. The achievable SE could be improved by increasing the bits of the phase shifters and existence of an optimal SNR and number of antennas.

Nimmagadda[33] applied modified Grey Wolf Optimization (GWO) referred as Improved Random Vector-based GWO (IRV-GWO) and modified Lion Algorithm (LA) referred as IRV-based LA (IRV-LA), to solve the complex optimization problems for beam-forming vectors and power allocation. This achieved better SE-EE tradeoff in massive MIMO technology.

Schmidt et al. [34] proposed a new architecture combining Tone-Reservation (TR) based Peak-to-Average Power Ratio (PAPR) reduction algorithm for massive MIMO system. The idea behind the proposed work was to obtain high precision beam-steering abilities, while maintaining the weighs assigned to each antenna, such that the Power Amplifier (PA) operate at the same Input Back-Off (IBO) leading to a higher EE. Implementation of the Beam Forming (BF) was proposed by adding progressive time delays to the signals fed to the antennas in the discrete time domain. The computational complexity was reduced significantly and a better performance/complexity tradeoff was provided. Good performance in BF was achieved while maintaining the low PAPR of the transmitted signals.

Liu et al. [35] studied the influence of the signal detection schemes on the EE of uplink MIMO systems with the low-resolution ADCs. The optimal power allocation and analytical approximations for ZF and ZF Successive Interference Cancellation (ZF-SIC) receivers were derived based on the assumption about equal transmission rates for all users. A large number of antennas were required to compensate for the loss due to the quantization errors while the number of BS antennas with the ZF-SIC receiver is significantly smaller than that with the $\mathrm{ZF}$ receiver. The ZF-SIC receiver was able to improve the overall EE for massive MIMO systems with practical ADCs.

Conventionally regular pilot was used in the MIMO system. It used the data and pilot symbols separately for the transmission purpose. Ragunathan and Perumal [36] proposed the superimposed pilots that overlays the data and pilot symbols for transmission. The proposed pilot allowed the usage of pilot symbols for a prolonged duration and reduced the pilot contamination. Pre-coding schemes such as MRC and ZF were used to analyze the performance of the pilots. The antenna selection algorithm was implemented with the precoding scheme, for performance improvement. The antennas with the threshold level were chosen to maximize the EE. The simulation results had proven that the superimposed pilot with the antenna selection algorithm resulted in the high EE than the random pilots. 
Khan et al. [37] investigated the EE and overall Power Transfer Efficiency (PTE) of a massive MIMO system. The harvested energy might be exploited by the users, to transmit information to the BS on the uplink. The overall system performance was analyzed while accounting for the nonlinear nature of the energy harvesters. A scalable model was used to characterize the PTE, for wireless energy transfer. The EE performance was characterized for the wireless transfer of energy and information. The optimal BS transmission power was derived in terms of the number of BS antennas and users. With the increase in the number of antennas and transmission power, the EE was improved to large number of antennas.

With the abundant increase in the contribution of SE and EE in massive MIMO technology, there are some challenges to improve the performance. Table 1 presents the disadvantages of the existing SE and EE models in massive MIMO system.

Table 1 Challenges of the existing SE and EE tradeoff models

\begin{tabular}{|c|c|}
\hline References & Challenges \\
\hline Fan and Zhang [38] & $\begin{array}{l}\text { - High computational load } \\
\text { - High complexity of matrix inversion }\end{array}$ \\
\hline Zhang et al. $[15,16]$ & - High computational complexity \\
\hline Li et al. [39] & $\begin{array}{l}\text { Long training interval depending on the coherence } \\
\text { intervals }\end{array}$ \\
\hline Huang et al. [40] & $\begin{array}{l}\text { Reaches a local minimum when error does not } \\
\text { change with increase in the number of iterations. }\end{array}$ \\
\hline He et al. [28] & - High computational complexity \\
\hline Patcharamaneepakorn et al. [29] & $\begin{array}{l}\text { - Analysis on heterogeneous network was } \\
\text { challenging } \\
\text { - Longer training duration resulted in the limitation } \\
\text { of the system performance }\end{array}$ \\
\hline Yang et al. [30] & $\begin{array}{l}\text { - More complex functioning } \\
\text { - Low performance gain }\end{array}$ \\
\hline Xin et al. [31] & $\begin{array}{l}\text { - Slow convergence rate } \\
\text { - Cannot detect multiple roots }\end{array}$ \\
\hline Tan et al. [32] & - Computationally expensive \\
\hline Nimmagadda[33] & $\begin{array}{l}\text { - Poor local searching capability } \\
\text { - Slow convergence rate }\end{array}$ \\
\hline Schmidt et al. [34] & $\begin{array}{l}\text { - The complexity of the TA-PAPR reduction } \\
\text { method is similar to the complexity of the FFT }\end{array}$ \\
\hline Lee $[41]$ & $\begin{array}{l}\text { - In some cases, while searching the optimum } \\
\text { parameters can require a lot of time consumption } \\
\text { with little performance improvement. } \\
\text { - Full rich scattering assumption is not always } \\
\text { valid for many situations }\end{array}$ \\
\hline You et al. [42] & $\begin{array}{l}\text { - Low convergence rate } \\
\text { - Increase in power consumption due to increase } \\
\text { in a greater number of antennas }\end{array}$ \\
\hline Jin et al. [43] & - Decrease in SE due to the channel correlation \\
\hline Liu et al. [35] & - Overall EE decreases for all receivers due to the \\
\hline
\end{tabular}




\begin{tabular}{|c|c|}
\hline & $\begin{array}{l}\text { strong pilot contamination and inter-cell } \\
\text { interference. } \\
\text { When practical coding and modulation schemes } \\
\text { are considered, decoding errors can result in } \\
\text { imperfect interference cancellation. }\end{array}$ \\
\hline Ragunathan and Perumal [36] & $\begin{array}{l}\text { - Consumption of more power compared to the } \\
\text { regular pilot }\end{array}$ \\
\hline Khan et al. [37] & $\begin{array}{l}\text { - Once the harvesters get saturated, the excess } \\
\text { power only increases the power consumption } \\
\text { without bringing any improvement in the } \\
\text { achievable rate }\end{array}$ \\
\hline
\end{tabular}

Lot of researches has focused on designing and analyzing the massive MIMO technology by solving the problem pertaining to the SE and EE. However, those models were intended to concentrate on only one parameter, i.e. either SE or EE. In order to develop an effective and robust design with balanced SE and EE, the Resource Efficiency (RE) metric considering the multi-objective function can be defined. Existing methods suffer due to the high computational complexity and slow convergence rate. Hence, a CSO algorithm is applied for channel optimization.

The main objective of the proposed model is to improve or enhance the RE function by optimizing the beam-forming vectors and power allocation for all the users.In the proposed work, the parameters and population are initialized and the fitness function is estimated. The optimal solutions are obtained and the positions of the hens, roosters and chickens are updated. The global optimal positions are obtained and then it is checked whether the maximum number of iterations are reached. If the maximum number of iterations is reached, the optimal channel output is obtained. Else, the process is returned to the estimation of the fitness function.

The proposed channel selection scheme initially estimates the channel using the received pilot signal in the sub-channel domain and interpolates the estimated channel to the frequency-spreading domain. Then, despreading of the channel compensated FS domain pilot is performed again to modify the Channel State Information (CSI) estimation. The transmitted data can be reconstructed after performing equalization in the frequency domain for each frequency point and frequency despreading process. The channel estimation is performed by the following process. At first, the estimated CSI at the pilot sub-channel location can be extracted after the despreading process and pilot extraction process. This CSI is interpolated for each frequency point and used for equalization of the received samples. Finally, the CSI for pilot subchannel is modified. These processes are performed in an iterative manner for a predefined number of times.

\section{Proposed Method}

\section{Channel model}

In the proposed work, the uplink of a single cell multi-user massive MIMO system is considered. In the MIMO system, there are ' $N$ ' number of antennas in a base station (BS). The BS receives data from ' $M$ ' single-antenna users in the same frequency resource. The data transmission is corrupted by the channel impairments. The data transmission from $\mathrm{M}$ users to the BS suffers from independent Rayleigh fading caused by multi-path reception and 
lognormal shadowing caused due to the presence of obstructions in the transmission path. The $N \times 1$ received signal vector at the BS is described as

$V=\sqrt{P_{T}} C_{X} X+\mathcal{N}$

Where ' $\mathrm{C}$ ' denotes the $N \times M$ channel matrix between the ' $\mathrm{M}$ ' number of single-antenna users and ' $\mathrm{N}$ ' number of BS antennas, $P_{T}$ represents the average transmission power of a single user in the channel, ' $\mathrm{X}$ ' indicates the vector of the symbols simultaneously transmitted by ' $\mathrm{M}$ ' single-antenna users and ' $\mathcal{N}$ ' denotes the noise vector.

As the BS antennas are located closely with each other, the large-scale fading for a single user across ' $\mathrm{N}$ ' number of BS antennas is correlated. But, the small-scale facing coefficients are independent and identically distributed. It is assumed that there is a perfect correlation between the shadowing components of a single user across ' $\mathrm{N}$ ' number of BS antennas. Hence, the received signals from the $M^{\text {th }}$ user across ' $N$ ' number of BS antennas suffer identical shadowing. The channel matrix is given as

$C=L D^{1 / 2}$

Where ' $\mathrm{L}$ ' is the $N \times M$ matrix of the small-scale fading coefficients and ' $\mathrm{D}$ ' is a $M \times M$ diagonal matrix including the large-scale fading coefficients of ' $M$ ' users. With the usage of a linear detector, the received signal is processed as

$R=\mathcal{A}^{O} \mathcal{Y}$

The vector ' $R$ ' represents the received signals from all the users, where $\mathcal{A}$ denotes the linear detector matrix depending on the channel matrix and ' $\mathrm{O}$ ' is the Hermitian operator.

\section{SINR Formulation}

After applying the linear detector, the received signal vector is given as

$R=\sqrt{P_{T}} \mathcal{A}^{O} C_{X}+\mathcal{A}^{O} \mathcal{N}$

The received signal vector is divided into two parts, to formulate the SINR of a single user. Let $R_{j}$ denote the received signal and $T_{j}$ indicate the transmitted symbol of the $\mathrm{j}^{\text {th }}$ user. Then,

$R_{j}=\sqrt{P_{T}} a_{j}^{O} C_{j}$

$T_{j}=\sqrt{P_{T}} \sum_{m=1, m \neq j}^{M} a_{j}^{O} C_{M} X_{M}+a_{j}^{O} \mathcal{N}$

Where $a_{j}$ and $C_{j}$ indicate the $\mathrm{j}^{\text {th }}$ columns of the linear detector matrix and channel matrix. The first term in the above equation denote the desired signal of the $\mathrm{j}^{\text {th }}$ user and other terms constitute the interference from other users and noise. Without the loss of generality, unit power spectral density of noise is assumed. The SINR of the $\mathrm{j}^{\text {th }}$ user can be represented as

$\operatorname{SINR}_{j}=\frac{P_{T}\left|a_{j}^{O} C_{j}\right|^{2}}{\sqrt{P_{T}} \sum_{m=1, m \neq j}^{M}\left|a_{j}^{O} C_{M}\right|^{2}+\left\|a_{j}\right\|^{2}}$

\section{Receiver Design}

A linear receiver is designed with the Maximum Ratio Combining (MRC) receiver combined with the Coordinate Descent Method (CDM) based algorithmic framework.

In the case of perfect CSI, the $N \times M$ linear detector matrix $\mathcal{A}$ for an MRC receiver is given by the channel matrix. The SINR of a single user for an MRC receiver is obtained as

$\operatorname{SINR}_{j}^{M R C}=\frac{P_{T}\left\|s_{j}\right\|^{4} L_{j}^{2}}{P_{T} L_{j} \Sigma_{m=1, m \neq j}^{M}\left|S_{j}^{O} S_{M}\right|^{2} L_{M}+\left\|S_{j}\right\|^{2} L_{j}}$

Conditioned on the small-scale fading coefficient for $\mathrm{j}^{\text {th }}$ user, a new Random Variable (RV) $C_{M}$ is defined such that $C_{M}=\frac{\left|s_{j}^{O} s_{M}\right|}{\left\|s_{j}\right\|}$ is a Gaussian RV with zero mean and unit variance 
independent of the small-scale fading coefficient for $\mathrm{j}^{\text {th }}$ user. Hence, $C_{M} \sim C N(0,1)$. The SINR is described as

$S I N R_{j}^{M R C}=\frac{P_{T}\left\|s_{j}\right\|^{2} L_{j}}{P_{T} \sum_{m=1, m \neq j}^{M}\left|C_{M}\right|^{2} L_{M}+1}$

Now, the Probability Density Function (PDF) of the SNR is derived. In the above equation, the numerator is the SNR ' $Z$ ' of a single user at the BS. Hence,

$Z=P_{T} L \sum_{I=1}^{M}\left|S_{I}\right|^{2}:=P_{T} L \gamma$

Where $\gamma \sim \Delta(N, 1)$ owing to the sum of the independent and identically distributed exponential RVs each having unit mean value.

From the above equation, it is clearly evident that the SNR follows a gamma-log normal product distribution. The PDF of a gamma RV is given by

$P_{C}(\gamma)=\frac{\gamma^{N-1} \exp (-\gamma)}{\Delta(N)}$

Where $\Delta(N)=(N-1)$ ! Since $\mathrm{N}$ is an integer. The distribution of a product $\mathrm{RV}, Z=L C$ is given by

$P_{Z}(Z)=\int_{-\infty}^{\infty} P_{L}(L) P_{C}\left(\frac{Z}{L}\right) \frac{1}{(L)} d L$

Since $P_{I}$ is a constant, it is neglected in the PDF expression of gamma log normal product distribution. From the above equation, the PDF of the product of gamma and log normal RVs is given by

$P_{Z}(Z)=\frac{\varphi Z^{N-1}}{(N-1) ! \sigma_{d B} \sqrt{2 \pi}} \int_{0}^{\infty} \frac{\exp (-z / L)}{L^{(N+1)}} \exp \left(\frac{-\left(\varphi \log _{e} L-\sigma_{d B}^{2}\right)}{2 \sigma_{d B}^{2}}\right) d L$

From the above equation, it is noted that the PDF of the SNR does not exist in a closed-form.

\section{CDM-based Signal Detector}

The CDM optimizes one variable at a time while holding the other variable fixed at their most recently updated values. Normally, the optimization coordinate is chosen cyclically. But, the CDM is efficient when the sub-problems could be solved [44].

For a given $X_{-\mu} \triangleq\left[x_{1}, \ldots, x_{\mu-1}, x_{\mu+1}, \ldots, x_{U}\right]$, the optimal value of $x_{\mu}=\mathcal{A}_{\mu} e^{j \theta_{\mu}}$ that minimizes $\left\|Y-S_{x}\right\|^{2}$ is given by

$\mathcal{A}_{\mu}^{*}=\frac{\left|\varphi_{\mu}\right|}{\sum_{b=1}^{B}\left|o_{b \mu}\right|^{2}}$

$\theta_{\mu}^{*}=\arg \left(\varphi_{\mu}\right)$

$\varphi_{\mu} \triangleq \sum_{b=1}^{B} O_{b \mu}^{*}\left(Y_{b}-\sum_{L=1, L \neq \mu}^{U} O_{b L} \mathcal{A}_{L} e^{j \theta_{L}}\right)$

The optimal update problem can be written as

$\mathcal{A}_{\mu}^{*} e^{j \theta_{\mu}^{*}}=\arg \min \sum_{b-1}^{B}\left|Y_{B}-\sum_{L-1}^{U} O_{b L} \mathcal{A}_{L} e^{j \theta_{L}}\right|^{2}$

\section{CDM}

$\mathrm{CDM}$ is an iterative procedure that performs sequential global minimization with respect to a single coordinate or multiple coordinates. At each iteration ' $M$ ', an index $I_{M} \in\{1,2, \ldots, n\}$ is selected and the decision vector is updated for the approximate minimization of the objective function in the $I_{M}$ th coordinate [45-47]. CDM is an effective way for solving optimization of multi-variables. During each iteration, CDM performs optimization and updation of only one variable while assuming that there is no change in other variables. Also, there is a need to initialize all variables firstly, so that all the variables have initial values. While solving the $\alpha^{\text {th }}$ variable, the previous $(\alpha-1)$ variables are all updated and the subsequent variables are initial values, so that the $\alpha^{\text {th }}$ variable could be solved and updated. 
The optimal $U_{\alpha}$ for a given $V_{\alpha}=\left(U_{1}, \ldots, U_{\alpha-1}, U_{\alpha+1}, \ldots, U_{N}\right)^{T}$ is described as

$U_{\alpha}=\varphi\left(\sum_{i=1, i \neq \alpha}^{N} A_{\alpha, i} U_{i}\right)$

Where $\varphi(x)$ denotes the phase of a complex variable ' $\mathrm{x}$ ', such as

$\varphi(x)=\left\{\begin{array}{l}1, x=0 \\ \frac{x}{|x|}, x \neq 0,\end{array}\right.$

\section{Iterative CDM Algorithm}

Input: $\overline{\mathrm{H}}, \mathrm{V}, \beta, \mathrm{K}, \mathrm{M}, \mathrm{N}$

Step 1: $T_{0}=I_{N_{S}}, V^{(0)}=V$

Step 2: for $i=1$ to $K$ do

Step 3: $\quad$ for $n=1$ to $N$ do

Step 4: $\quad A=\overline{\mathrm{H}}^{H} T_{n-1}^{-1} \overline{\mathrm{H}}$

Step 5: $\quad$ for $\alpha=1$ to $M$ do

Step 6: $\quad$ Determine $U_{\alpha}$

Step 7: $\quad$ end for

Step 8: $\quad$ Update $V_{n}=\left[U_{1}, \ldots, U_{M}\right]^{T}$

Step 9: $\quad T_{n}=I_{N_{S}}+\beta \overline{\mathrm{H}} V_{n}^{(i)} V_{n}^{(i)^{H}} \overline{\mathrm{H}}^{H}$

Step 10: $\quad$ end for

Step 11: $\quad$ Update $V^{(i)}=\left[v_{1}, \ldots, v_{N}\right]$

Step 12: end for

Step 13: $V^{\text {out }}=V^{(K)}$

It is set that the stopping criterion triggers the iterative algorithm as the number of iterations. It is assumed that the channel matrix ' $\mathrm{H}$ ' is known at the transmitter. $\mathrm{H}$ can be estimated by the receiver and fed back to the transmitter. Hence, the receiver should first estimate the perfect channel information, calculate the digital precoder $F_{B B}$, digital combining matrix $W_{B B}$, analog precoder $F_{R F}$ and analog combining matrix $W_{R F}$ and fed back analog and digital precoders to the transmitter $[48,49]$.

\section{Combined Hybrid Precoding Algorithm by CDM}

Input: $\mathrm{H}, N_{R F}^{T}, N_{R F}^{R}, N_{S}$

Step 1: Construct Analog precoder and analog combining matrix with random phases

Step 2: Repeat

Step 3:

$\overline{\mathrm{H}}=W_{R F}^{H} H$

Step 4: $\quad$ Obtain analog precoder by solving the precoder and combiner design problem

Step 5: $\quad \overline{\mathrm{H}}=F_{R F}^{H} H^{H}$

Step 6: Obtain analog combining matrix by solving the precoder and combiner design problem using iterative CDM Algorithm

Step 7: Until a stopping criterion is achieved

Step 8: $H_{e}=W_{R F}^{H} H F_{R F}$ and its Singular Value Decomposition (SVD) is $H_{e}=U_{e} \sum_{e} V_{e}^{H}$

Step 9: $F_{B B}=V_{e}\left(:, 1: N_{S}\right), W_{B B}=U_{e}\left(:, 1: N_{S}\right)$

Step 10: $F_{B B}=\sqrt{N_{S}} \frac{F_{B B}}{\left\|F_{R F} F_{B B}\right\|_{F}^{2}}$ and $W_{B B}=\sqrt{N_{S}} \frac{W_{B B}}{\left\|W_{R F} W_{B B}\right\|_{F}^{2}}$ 

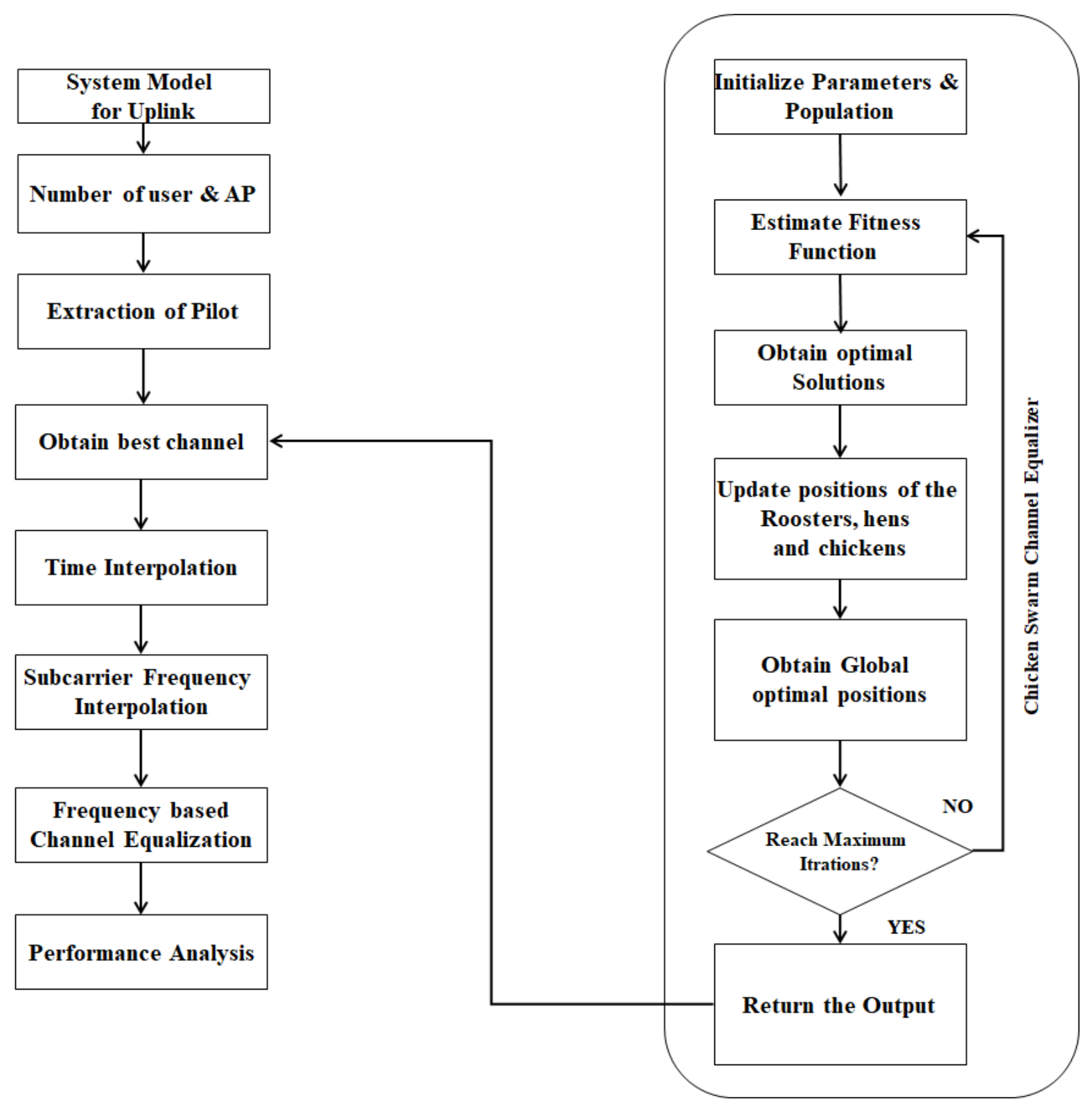

Figure 1 Flow diagram of the proposed CS scheme with CSO Algorithm

\section{Proposed Flow}

Figure 1 shows the flow diagram of the proposed work. The optimal power allocation, BF vector and optimization of the transmission power are implemented using the CSO algorithm. During the optimal power allocation, BF vectors are determined based on the following steps

1. Determine the BF vector that should satisfy the SINR constraints.

a. Check whether the computed BF vector satisfies the SINR constraints.

b. If not, optimize the BF vector with some percentage of alterations, such that the alteration length should be equal to the length of the obtained BF vector.

c. The objective function for generating the optimal BF vectors is satisfying the SINR constraints. The power penalty of 100 is given, if the SINR constraints are not satisfied.

d. The iteration is stopped, once the power penalty reaches zero. The best solution is taken as the optimal BF vector. 
2. The objective function for optimizing the transmission power is the maximization of the RE metric.

The solution with maximum RE metric is taken as the best transmission power. Thus, the massive MIMO system is obtained with better tradeoff between SE and EE.

Data group has to be spread over multiple frequency points, which correspond to the frequency coefficients of prototype filter $F_{|P|}$ for $(-P+1 \leq p \leq P-1)$. This process is called as frequency dispreading. Frequency despreading is applied to synchronize the input data in a frequency domain, to recover the original data. This makes the input data robust to the interference. After frequency spreading, all the frequency points are transmitted to generate a single symbol.

The received signal is expressed as follows

$R(t)=\sum_{x=0}^{X-1} \sum_{y=0}^{Y-1} D_{x, y} H_{x, y} \mathcal{G}_{x, y}(t)+W_{G}(t)$

Where ' $\mathrm{X}$ ' is the number of sub-channels and ' $\mathrm{Y}$ ' is the number of frequency points, $H_{x, y}$ is the transmission Channel State Information (CSI), $\mathcal{G}_{x, y}(t)$ is the filter of the $\mathrm{x}^{\text {th }}$ subchannel in the $\mathrm{y}^{\text {th }}$ frequency point and $W_{G}(t)$ denotes the white Gaussian noise. After matched filtering in the analysis filter bank, the result is represented as

$R_{x, y}=H_{x, y}\left(D_{x, y}+j \sum_{P, Q \neq x, y} D_{P, Q}\langle\mathcal{G}\rangle_{x, y}^{P, Q}\right)+W_{G_{x, y}}=H_{x, y}\left(D_{x, y}+I_{x, y}\right)+W_{G_{x, y}}$

If the scattered pilot sub-channel scheme is used for the estimation of CSI, the estimated CSI at the pilot location sub-channel $\left(x_{P}, y_{P}\right)$ is represented as follows [4]

$\widehat{H}_{x_{P}, y_{P}}=\frac{R_{x_{P}, y_{P}}}{D_{x_{P}, y_{P}}}=\frac{H_{x_{P}, y_{P}}\left(D_{x_{P}, y_{P}}+I_{x_{P}, y_{P}}\right)+W_{G_{x_{P}}, y_{P}}}{D_{x_{P}, y_{P}}}$

The transmitted data can be reconstructed after performing the equalization process in the frequency domain for each frequency point and frequency despreading process. Hence, subchannel CSI estimation for each frequency point is required. First, the CSI estimation for each subchannel can be obtained after the interpolation process. Then, the CSI for each frequency point can be obtained by interpolating again between the CSI of the neighboring subchannels.

In this case, the CSI for each subchannel at the center frequency point has to be obtained for the interpolation of other frequency points. If the transmission channel is flat during the subchannel, the CSI estimation is equivalent with the CSI at the center frequency point. Hence, the CSI for each subchannel is substituted directly for the center frequency point at first CSI interpolation.

But, if the transmission CSI cannot be considered as flat during each subchannel, this CSI for each subchannel cannot be ensured to be equivalent with the center frequency point. In this case, this estimated CSI has to be modified to improve the performance of channel equalization task. One way to check the reliability of estimated CSI is to compare the reproduced pilot at the receiver with the transmitted pilot signal. If the CSI is estimated perfectly for every frequency point in a pilot subchannel, the data part for a pilot subchannel is equivalent to the amplitude of the pilot symbol. 
The channel estimation scheme is performed by the following process. At first, the estimated CSI at the pilot subchannel location can be extracted after the frequency despreading and pilot extraction process. This CSI is interpolated for each frequency point, which is used for the equalization of the received samples.

The CSI for every stage is stored in the channel store/correction block, interpolated in the time and frequency interpolator to obtain the CSI about each frequency point. Then, the frequency equalization for each frequency point is performed in channel equalizer. After then, the CSI modification is performed at the channel store/modification block, with the pilot estimation value $[50,51]$. Then, the performance of the proposed CS scheme with the CSO algorithm is analyzed.

\section{CSO for optimal channel allocation}

Generally, the behavior of the chicken is based on its gender. The head roosters being a dominant one will search for the food and fight with the chickens who invade the territory. It crows louder when chickens from other groups invade their territory. The rooster will dominate the weak chickens and might call their group mates to share the food. The dominant hens remain near the roosters and submissive hens would stand at the periphery of the group in search of food. The chicks always stay around the mothers and search food over there. As a swarm, they all cooperate as a team/group to search food in a specific hierarchical order of roosters, hens and chicks.

Meng et al. [52] proposed a new bio-inspired algorithm, CSO algorithm that mimics the hierarchical order and behavior of the chicken swarm. The chicken swarm consists of several groups: dominant head roosters, couple of hens and chicks.The swarm is divided into multiple groups and identity of the chickens is determined depending on their fitness values. The chickens with best fitness values would act as a head rooster in a group. The chickens with worst several fitness values would act as chicks. Others would be the hens that randomly choose which group to live in. The mother-child relationship between the hens and the chicks is also randomly established. The hierarchal order, dominant and hen-chick relationships in a group will remain unchanged. Chickens follow the head rooster of their own group to search for food, while preventing the ones from eating their own food. It is assumed that the chickens would randomly steal the good food already found by others. The chicks search for food around their mother hens. The dominant roosters always have advantage in competition for the food.

Let us assume that ' $N_{R}$ ', ' $N_{H}$ ', ' $N_{C}$ ' and ' $N_{M}$ ' denote the number of the roosters, hens, chicks and the mother hens in a group, respectively. The best $N_{R}$ would be assumed to be the roosters, while the worst $N_{C}$ ones would be the chicks. The rest are treated as hens. All ' $\mathrm{M}$ ' virtual chickens represented by their positions $X_{a, b}^{t}(a \in[1, \ldots, M], b \in[1, \ldots, S])$ at the time step ' $t$ ', search for the food in the ' $\mathrm{S}$ ' dimensional space.

\section{Chicken Movement}

The roosters with the best fitness values have priority for the food access than the ones with the worst fitness values. This case can be simply simulated by the situation that the roosters with better fitness values can search for food in a wider range of places when compared to the roosters with worse fitness values. This is described using the following equation

$$
P_{a, b}^{t+1}=P_{a, b}^{t} *\left[1+G\left(0, \sigma^{2}\right)\right]
$$


Where $\sigma^{2}=\left\{\begin{array}{c}1, \text { if } F_{a} \leq F_{I} \\ \exp \left[\frac{\left(F_{I}-F_{a}\right)}{\left|F_{a}\right|+\psi}\right], \text { otherwise }\end{array} I \in[1, N], I \neq a\right.$

Where $G\left(0, \sigma^{2}\right)$ is the Gaussian distribution with mean 0 and standard deviation $\sigma^{2} . \psi$ is used to avoid the zero division error. 'I' is the rooster's index that is randomly selected from the group of roosters. ' $\mathrm{F}$ ' is the fitness value of the corresponding position ' $\mathrm{P}$ '.

The hens could follow their group-mate roosters in search of food. They can also steal the good found by other chickens, though oppressed by other chickens. The dominant hens have the advantage of competing for food than the submissive hens. This is mathematically expressed as below

$P_{a, b}^{t+1}=P_{a, b}^{t}+Q_{1} * U_{R} *\left(P_{I_{R}, b}^{t}-P_{a, b}^{t}\right)+Q_{2} * U_{R} *\left(P_{I_{C}, b}^{t}-P_{a, b}^{t}\right)$

Where $Q_{1}=\exp \left[\left(F_{a}-F_{I_{R}}\right) /\left(a b s\left(F_{a}\right)+\psi\right)\right]$

$Q_{2}=\exp \left[\left(F_{I_{C}}-F_{a}\right)\right]$

Where $U_{R}$ represents the uniform random number over $[0,1], I_{R} \in[1, \ldots, N]$ denotes the index of the rooster which is the group-mate of $a^{\text {th }}$ hen. $I_{C} \in[1, \ldots, N]$ indicates the index of the chicken which is randomly chosen from the swarm. $I_{R} \neq I_{C}$.

It is obvious that $F_{a}>F_{I_{R}}$ and $F_{a}>F_{I_{C}}$ and hence $Q_{2}<1<Q_{1}$. If it is assumed that $Q_{1}=0$, then the $\mathrm{a}^{\text {th }}$ hen would search for the food followed by other chickens. Bigger the difference between the fitness values of two chickens, the smaller $Q_{2}$ value and larger gap between the positions of two chickens. Thus, the hens would not easily steal the food found by other chickens. The difference in the formula of $Q_{2}$ from $Q_{1}$ is due to the existence of competitions in a group. If $Q_{2}=0$, then the $\mathrm{a}^{\text {th }}$ hen would search for the food in their own territory. The fitness value of the rooster is unique for the specific group. The smaller the fitness value of $\mathrm{a}^{\text {th }}$ hen, nearer is the approximation of $Q_{1}$ to 1 and smaller is the gap between the positions of the $\mathrm{a}^{\text {th }}$ hen and its group-mate rooster. Henceforth, the more dominant hens are more likely to eat the food than the submissive ones. The chicks would move around their mother hens in search of food.

$P_{a, b}^{t+1}=P_{a, b}^{t}+F L *\left(P_{M, b}^{t}-P_{a, b}^{t}\right)$

Where $P_{M, b}^{t}$ denotes the position of the mother of a a $^{\text {th }} \operatorname{chick}(M \in[1, N])$. FL $(F L \in(0,2))$ is a parameter denoting that the chick would follow the mother hen in search of food.

The Mean Square Error (MSE) between the estimated CSI and original channel CSI is computed at all pilot subchannels. The estimation of CSI is affected by the number of subchannels and frequency selection. The CSI estimation performance is improved with the increase in the number of iterations.

\section{CSO Algorithm}

Initialization of population of ' $\mathrm{N}$ ' chickens and passing the weights to the network

Evaluate the fitness values of ' $N$ ' chickens, $F=0$;

Loading the training data to the network

While MSE<Stopping Criteria

If $(F \%$ Gen $==0)$ 
Rank the fitness values of the chickens and establish a hierarchical order in the swarm;

Divide the swarm into various groups, and determine the relationship between the End If mother hens and chicks in a group;

For $\mathrm{i}=1: \mathrm{N}$

If $i==$ rooster

Update its location;

End If

If $i==$ hen

Update its location;

End If

If $i==$ chick

Update its location;

End If

Evaluate the new solution;

If the new solution is better than the previous one, replace the previous solution with the new solution;

End For

Swarm finds the best weights and passes to the network;

Chicken keeps on calculating the best possible weight at each epoch until the convergence of network;

End While

\section{Results}

Simulation of the proposed algorithm is done using MATLAB 2014a. The simulation parameters are described in Table 2. The performance of the proposed Channel Selection (CS) with CSO algorithm is evaluated in terms of SE and EE by comparing with Fully Connected-Zero Forcing (FC-ZF) Precoding, Hybrid Precoding [53], Quantized Hybrid Precoding [54], B-MIMO Precoding [55] and CSO-Dynamic Phase Adjustment (DPA) [56]. Figure 2 shows the comparison of the FC-ZF Precoding, Hybrid Precoding, Quantized Hybrid Precoding and B-MIMO Precoding. From the graph, it is found out that the SE increases with the increase in the SNR. By choosing the optimal channel for data transmission, the proposed scheme yields better performance than the conventional algorithms.

Table 2 Simulation Parameters

\begin{tabular}{|c|c|}
\hline Parameters & Value \\
\hline Radius of cell & $1000 \mathrm{~m}$ \\
\hline Reference distance & $100 \mathrm{~m}$ \\
\hline Path-loss exponent & 3.8 \\
\hline Shadow fading standard deviation & $8 \mathrm{~dB}$ \\
\hline Target BER & $10^{-3}$ \\
\hline Number of antennas in BS & 128 \\
\hline Number of users & 4 \\
\hline Estimation error variance & 0.1 \\
\hline Noise power & $-104 \mathrm{dBm}$ \\
\hline Power amplifier efficiency & 0.3 \\
\hline
\end{tabular}




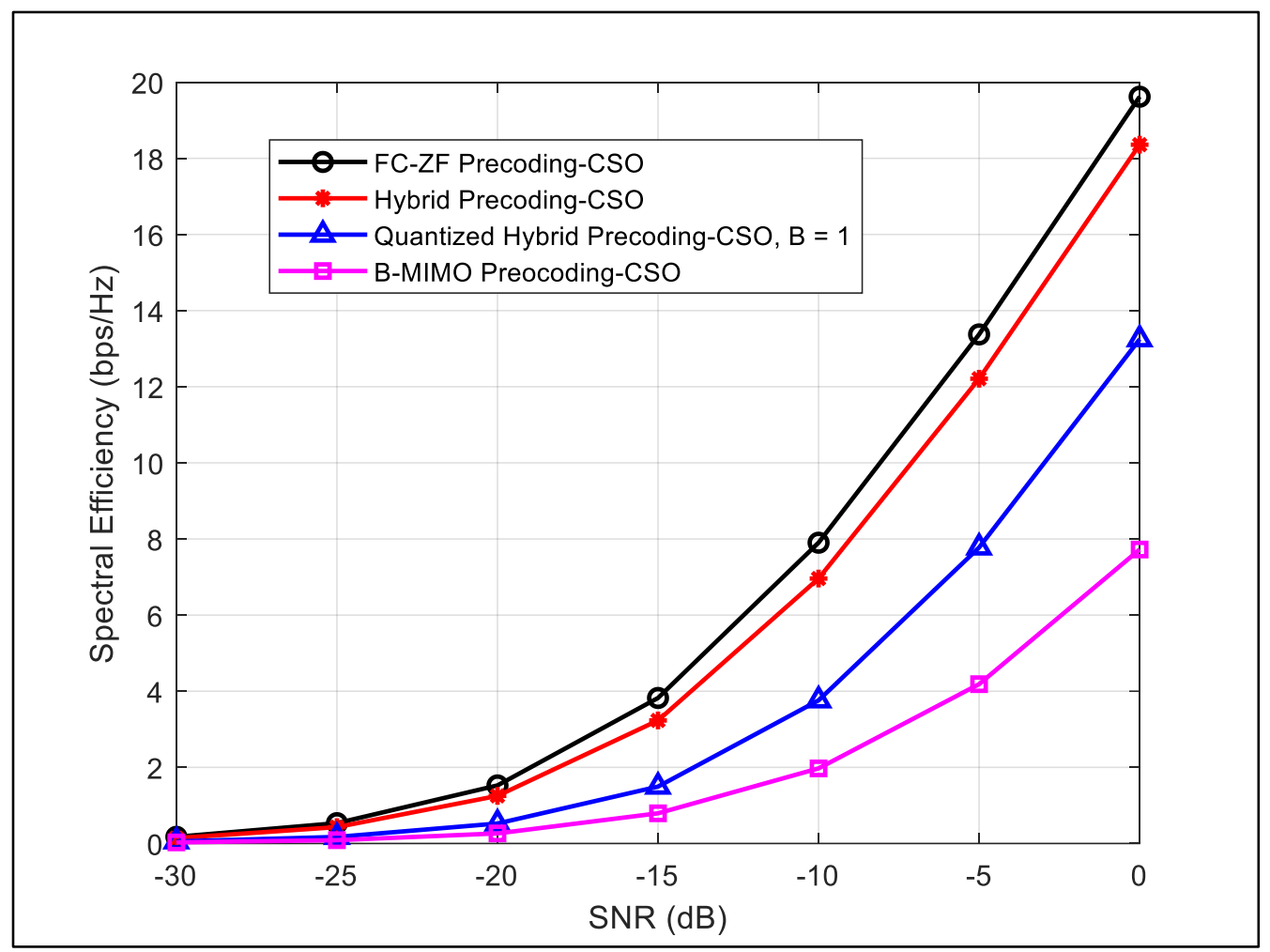

Figure 2 SE analysis of FC-ZF Precoding, Hybrid Precoding, Quantized Hybrid Precoding and B-MIMO Precoding

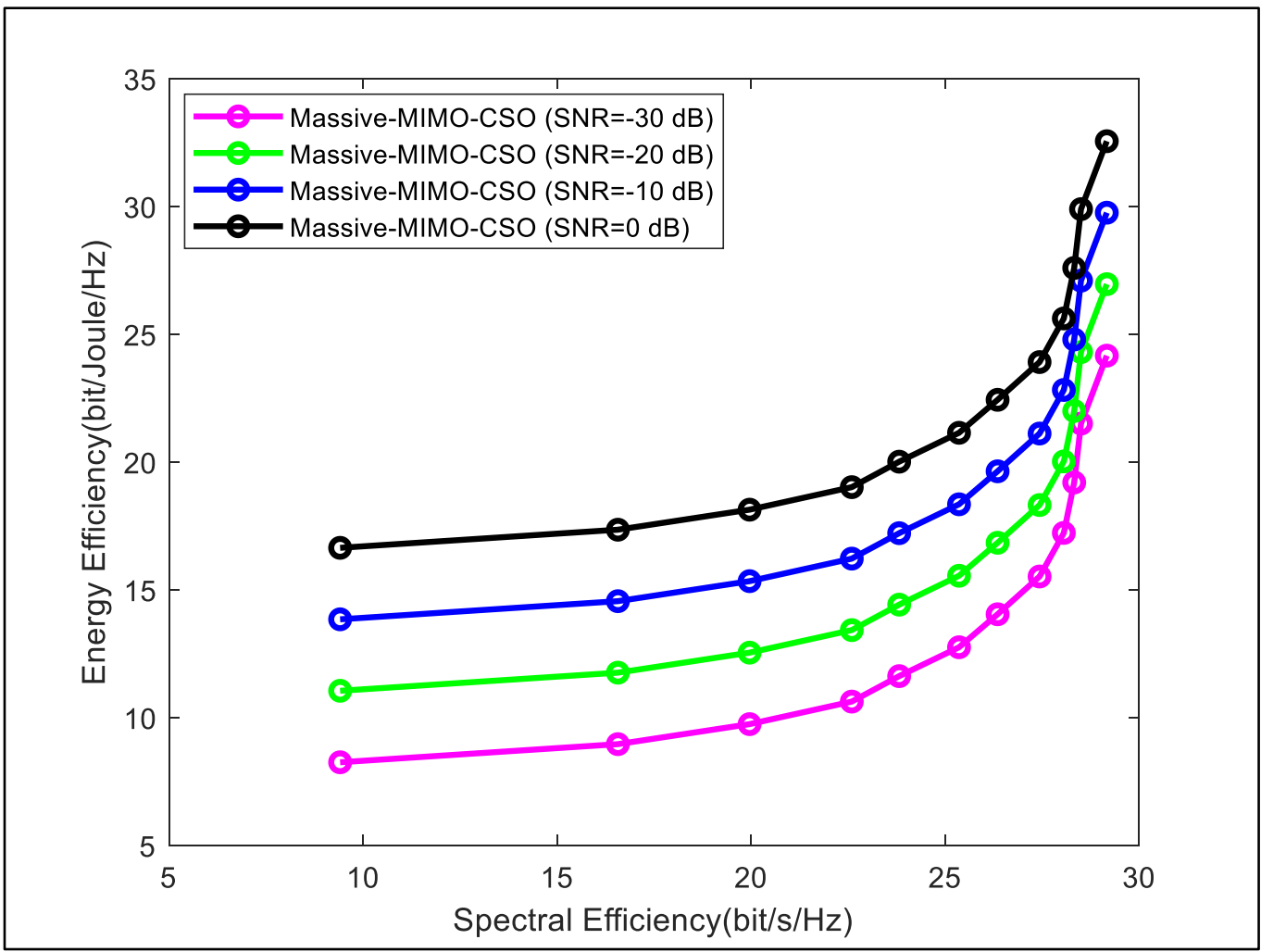

Figure 3 Comparative analysis of spectral and energy efficiencies for the proposed massive MIMO-CSO 
Figure 2 shows the analysis of Spectral efficiency for different precoding techniques. Figure 3 illustrates the comparative analysis of SE and EE for the proposed MIMO-CSO for various SNR values. It is observed that the SE increases with the increase in EE. The tradeoff between SE and EE is balanced by optimizing the power allocation and beam-forming vectors using the CSO algorithm.

The proposed massive MIMO-CSO is compared with the existing near and sub-optimal schemes [57]. Figure 4 illustrates the EE Analysis of existing near and sub-optimal schemes and proposed massive MIMO-CSO for different number of users. The number of users in our proposed scheme is 4. The near and sub-optimal schemes obtain similar EE performance with low complexity. The proposed massive MIMO-CSO achieves higher EE than the near and sub-optimal schemes.

Figure 5 presents the SE Analysis of existing near and sub-optimal schemes and proposed massive MIMO-CSO for different number of BS antennas. The SE performance of the massive MIMO system is improved with the increase in the number of BS antennas as it brings greater spatial diversity gain. It is observed that the near-optimal scheme and suboptimal schemes have similar SE with lower complexity. Especially for larger number of BS antennas, performance of the proposed massive MIMO-CSO is better than the near and suboptimal schemes.

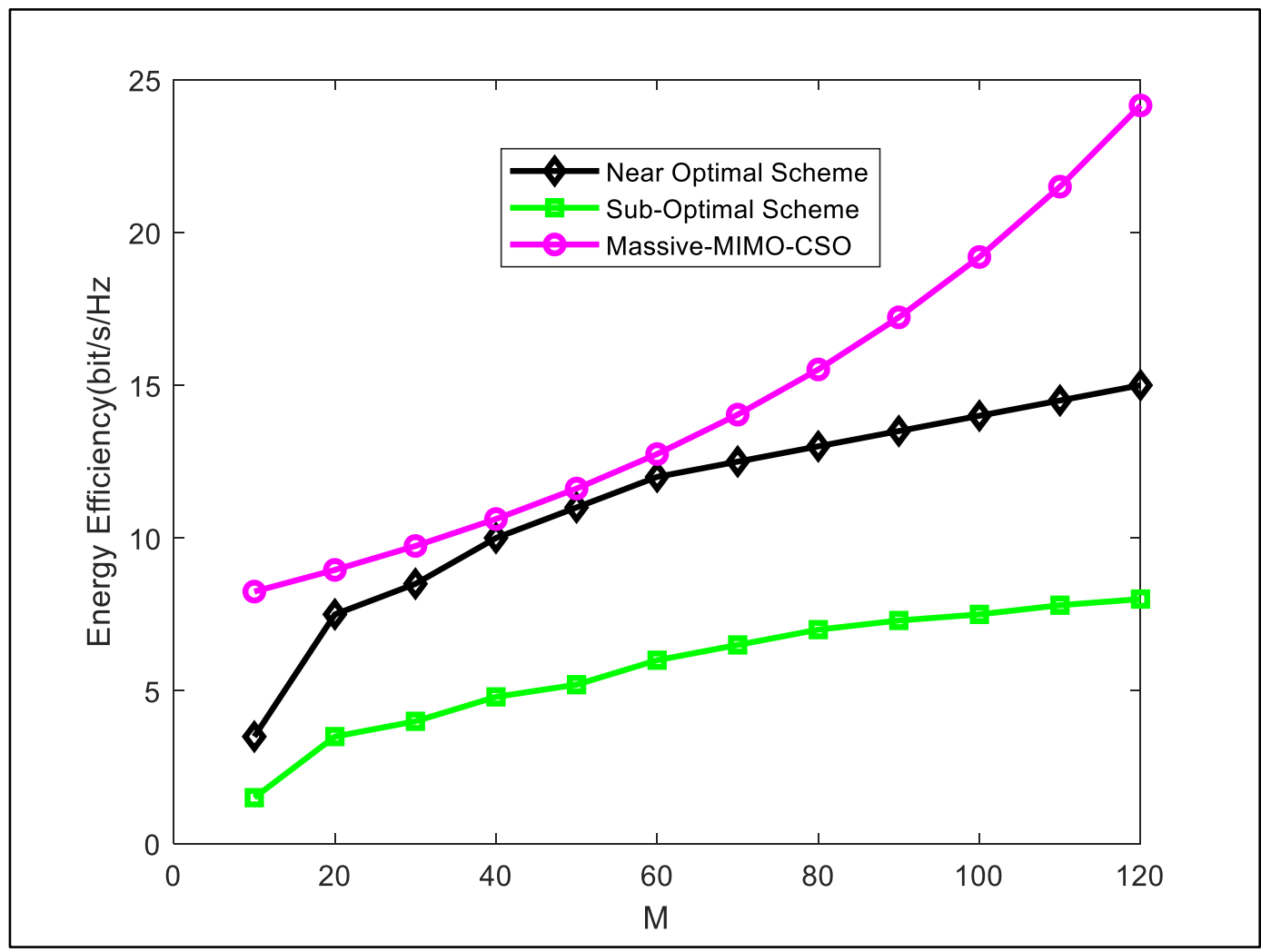

Figure 4 EE Analysis of existing near and sub-optimal schemes and proposed massive MIMO-CSOfor different number of users 


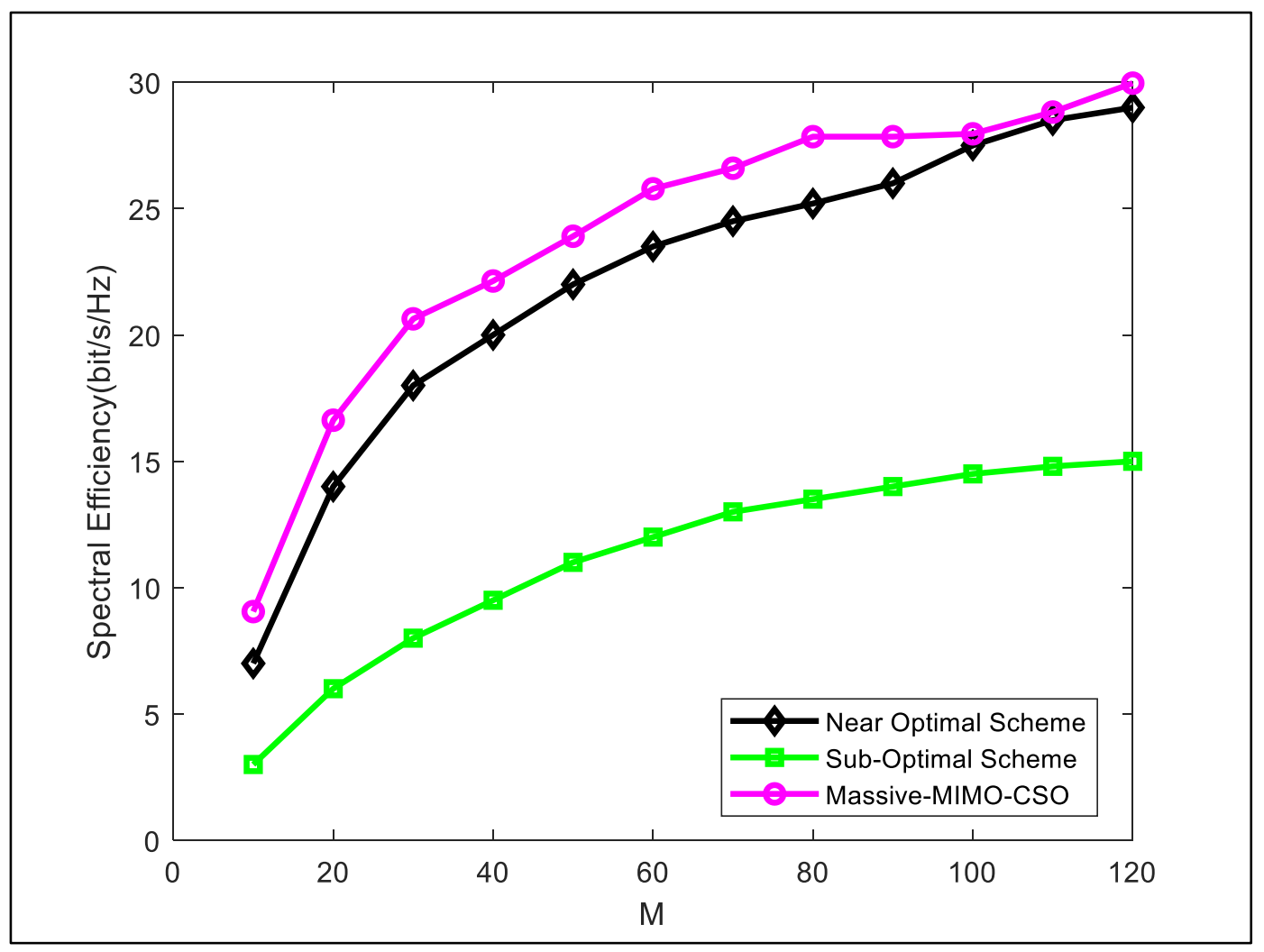

Figure 5 Comparative SE analysis of the proposed massive MIMO-CSO scheme and existing near and sub optimal schemes

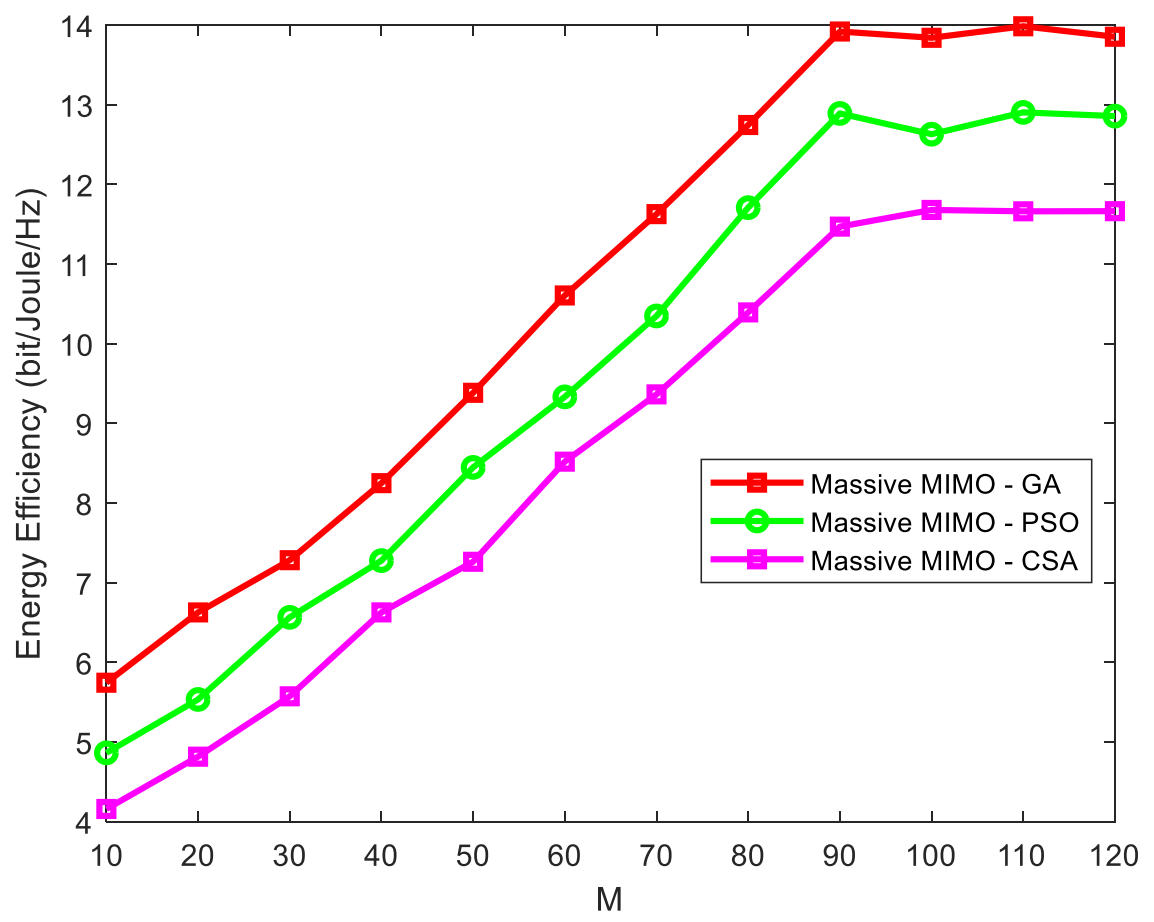

Figure 6 Comparative analysis of EE of GA, PSO and CSO algorithms 


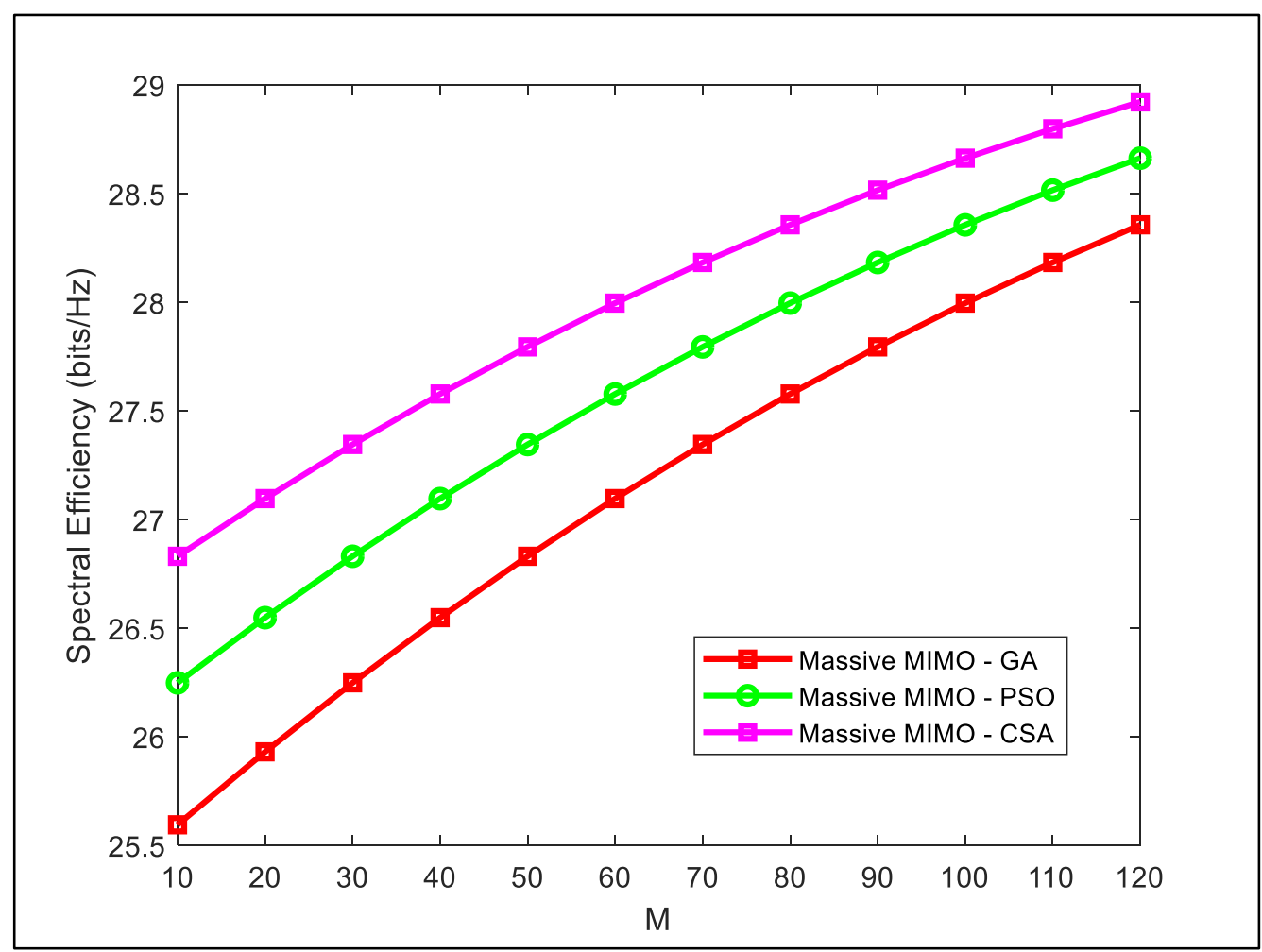

Figure 7 Comparative analysis of SE of GA, PSO and CSO algorithms

Figure 6 depicts the comparative analysis of the energy efficiency of the GA, PSO and CSO algorithms. Figure 9 presents the SE analysis of the GA, PSO and CSO algorithms. From the graphs, it is observed that the proposed CSO algorithm yields better SE and EE than the existing GA and PSO algorithms.

\section{Conclusion}

In the proposed work, the trade-off between SE and EE of the massive MIMO is balanced using the bio-inspired CSO algorithm. The main objective of the proposed scheme is to find the optimal solution for beam-forming vectors and power allocation and an optimal channel for the data transmission. The optimal solution has been found to attain maximum SE and EE through the RE metric model. CSO algorithm is applied to find the optimal channel for transmission. The channel state information is predicted and a projection matrix with channel estimation framework is formed. The selection of the index sets in the iteration process provides the optimized channel. Data transmission is performed through the optimal channel. From the experimental results, it is concluded that the proposed CS scheme with the CSO algorithm yields better SE and EE than the existing algorithms.

\section{Declarations}

Funding: The authors receive no financial support for the research or publication of this article.

Conflict of interest: The authors declare no conflicts of interest to publish this paper.

Availability of data and material: Data sharing is not applicable as no datasets were generated or analyzed during this study

Code availability: The code generated during this study is available with the corresponding author

Ethics approval: This research does not involve human or animal participants.

Consent to participate: Not applicable 
Consent for publication: Not applicable.

\section{References}

[1] W. Tan, S. Jin, C.-K. Wen, and T. Jiang, "Spectral efficiency of multi-user millimeter wave systems under single path with uniform rectangular arrays," EURASIP Journal on Wireless Communications and Networking, vol. 2017, no. 1, p. 181, 2017.

[2] L. Fan, R. Zhao, F.-K. Gong, N. Yang, and G. K. Karagiannidis, "Secure multiple amplifyand-forward relaying over correlated fading channels," IEEE Transactions on Communications, vol. 65, no. 7, pp. 2811-2820, 2017.

[3] H. Q. Ngo, E. G. Larsson, and T. L. Marzetta, "Energy and spectral efficiency of very large multiuser MIMO systems," IEEE Transactions on Communications, vol. 61, no. 4, pp. 14361449, 2013.

[4] T. L. Marzetta, "Noncooperative cellular wireless with unlimited numbers of base station antennas," IEEE transactions on wireless communications, vol. 9, no. 11, pp. 3590-3600, 2010.

[5] L. Lu, G. Y. Li, A. L. Swindlehurst, A. Ashikhmin, and R. Zhang, "An overview of massive MIMO: Benefits and challenges," IEEE journal of selected topics in signal processing, vol. 8, no. 5, pp. 742-758, 2014.

[6] E. G. Larsson, O. Edfors, F. Tufvesson, and T. L. Marzetta, "Massive MIMO for next generation wireless systems," IEEE communications magazine, vol. 52, no. 2, pp. 186-195, 2014.

[7] F. Rusek et al., "Scaling up MIMO: Opportunities and challenges with very large arrays," IEEE signal processing magazine, vol. 30, no. 1, pp. 40-60, 2012.

[8] G. Pan et al., "On secrecy performance of MISO SWIPT systems with TAS and imperfect CSI," IEEE Transactions on Communications, vol. 64, no. 9, pp. 3831-3843, 2016.

[9] A. He, L. Wang, M. Elkashlan, Y. Chen, and K.-K. Wong, "Spectrum and energy efficiency in massive MIMO enabled HetNets: A stochastic geometry approach," IEEE Communications Letters, vol. 19, no. 12, pp. 2294-2297, 2015.

[10] B. Murmann, "ADC performance survey 1997-2016," Online] http://www. stanford. edu/murmann/adcsurvey. html, 2017.

[11] C. Risi, D. Persson, and E. G. Larsson, "Massive MIMO with 1-bit ADC," arXiv preprint arXiv:1404.7736, 2014.

[12] J. Choi, J. Mo, and R. W. Heath, "Near maximum-likelihood detector and channel estimator for uplink multiuser massive MIMO systems with one-bit ADCs," IEEE Transactions on Communications, vol. 64, no. 5, pp. 2005-2018, 2016.

[13] Y. Li, C. Tao, G. Seco-Granados, A. Mezghani, A. L. Swindlehurst, and L. Liu, "Channel estimation and performance analysis of one-bit massive MIMO systems," IEEE Transactions on Signal Processing, vol. 65, no. 15, pp. 4075-4089, 2017.

[14] C. Mollen, J. Choi, E. G. Larsson, and R. W. Heath, "Uplink performance of wideband massive MIMO with one-bit ADCs," IEEE Transactions on Wireless Communications, vol. 16, no. 1, pp. 87-100, 2016.

[15] J. Zhang, L. Dai, S. Sun, and Z. Wang, "On the spectral efficiency of massive MIMO systems with low-resolution ADCs," IEEE Communications Letters, vol. 20, no. 5, pp. 842-845, 2016.

[16] Z. Zhang, Z. Chen, M. Shen, and B. Xia, "Spectral and energy efficiency of multipair twoway full-duplex relay systems with massive MIMO," IEEE journal on selected areas in communications, vol. 34, no. 4, pp. 848-863, 2016.

[17] J. Zhang, L. Dai, Z. He, S. Jin, and X. Li, "Performance analysis of mixed-ADC massive MIMO systems over Rician fading channels," IEEE Journal on Selected Areas in Communications, vol. 35, no. 6, pp. 1327-1338, 2017.

[18] X. Chen, X. Wang, and X. Chen, "Energy-efficient optimization for wireless information and power transfer in large-scale MIMO systems employing energy beamforming," IEEE Wireless Communications Letters, vol. 2, no. 6, pp. 667-670, 2013. 
[19] J.-H. Lee and J.-Y. Lee, "Optimal beamforming-selection spatial precoding using populationbased stochastic optimization for massive wireless MIMO communication systems," Journal of the Franklin Institute, vol. 354, no. 10, pp. 4247-4272, 2017.

[20] S. Todnatee and C. Phongcharoenpanich, "Iterative GA optimization scheme for synthesis of radiation pattern of linear array antenna," International Journal of Antennas and Propagation, vol. 2016, 2016.

[21] M. M. Khodier and M. Al-Aqeel, "Linear and circular array optimization: A study using particle swarm intelligence," Progress In Electromagnetics Research, vol. 15, pp. 347-373, 2009.

[22] P. Saxena and A. Kothari, "Ant lion optimization algorithm to control side lobe level and null depths in linear antenna arrays," AEU-International Journal of Electronics and Communications, vol. 70, no. 9, pp. 1339-1349, 2016.

[23] U. Singh and R. Salgotra, "Synthesis of linear antenna array using flower pollination algorithm," Neural Computing and Applications, vol. 29, no. 2, pp. 435-445, 2018.

[24] S. K. Mahto and A. Choubey, "A novel hybrid IWO/WDO algorithm for interference minimization of uniformly excited linear sparse array by position-only control," IEEE Antennas and Wireless Propagation Letters, vol. 15, pp. 250-254, 2015.

[25] S. Jayaprakasam, S. K. A. Rahim, and C. Y. Leow, "PSOGSA-Explore: A new hybrid metaheuristic approach for beampattern optimization in collaborative beamforming," Applied Soft Computing, vol. 30, pp. 229-237, 2015.

[26] G. Sun, Y. Liu, J. Zhang, A. Wang, and X. Zhou, "Node selection optimization for collaborative beamforming in wireless sensor networks," Ad Hoc Networks, vol. 37, pp. 389403, 2016.

[27] Y. Li, C. Tao, L. Liu, G. Seco-Granados, and A. L. Swindlehurst, "Channel estimation and uplink achievable rates in one-bit massive MIMO systems," in 2016 IEEE Sensor Array and Multichannel Signal Processing Workshop (SAM), 2016: IEEE, pp. 1-5.

[28] A. He, L. Wang, Y. Chen, K.-K. Wong, and M. Elkashlan, "Spectral and energy efficiency of uplink D2D underlaid massive MIMO cellular networks," IEEE Transactions on Communications, vol. 65, no. 9, pp. 3780-3793, 2017.

[29] P. Patcharamaneepakorn, S. Wu, C.-X. Wang, M. M. Alwakeel, X. Ge, and M. Di Renzo, "Spectral, energy, and economic efficiency of 5G multicell massive MIMO systems with generalized spatial modulation," IEEE Transactions on Vehicular Technology, vol. 65, no. 12, pp. 9715-9731, 2016.

[30] J. Yang, H. Wang, J. Ding, X. Gao, and Z. Ding, "Spectral and energy efficiency analysis for massive MIMO multi-pair two-way relaying networks under generalized power scaling," Science China Information Sciences, vol. 60, no. 10, p. 102303, 2017.

[31] Y. Xin, D. Wang, J. Li, H. Zhu, J. Wang, and X. You, "Area spectral efficiency and area energy efficiency of massive MIMO cellular systems," IEEE Transactions on Vehicular Technology, vol. 65, no. 5, pp. 3243-3254, 2015.

[32] W. Tan, D. Xie, J. Xia, W. Tan, L. Fan, and S. Jin, "Spectral and energy efficiency of massive MIMO for hybrid architectures based on phase shifters," IEEE Access, vol. 6, pp. 1175111759, 2018.

[33] S. M. Nimmagadda, "Optimal spectral and energy efficiency trade-off for massive MIMO technology: analysis on modified lion and grey wolf optimization," Soft Computing, pp. 1-17, 2020.

[34] C. A. Schmidt, M. Crussière, J. F. Hélard, and A. M. Tonello, "Improving energy efficiency in massive MIMO: joint digital beam-steering and tone-reservation PAPR reduction," IET Communications, vol. 14, no. 14, pp. 2250-2258, 2020.

[35] T. Liu, J. Tong, Q. Guo, J. Xi, Y. Yu, and Z. Xiao, "Energy efficiency of massive MIMO systems with low-resolution ADCs and successive interference cancellation," IEEE Transactions on Wireless Communications, vol. 18, no. 8, pp. 3987-4002, 2019.

[36] S. Ragunathan and D. Perumal, "Enhancement of energy efficiency in massive MIMO network using superimposed pilots," Journal of Ambient Intelligence and Humanized Computing, pp. 1-8, 2020. 
[37] T. A. Khan, A. Yazdan, and R. W. Heath, "Optimization of power transfer efficiency and energy efficiency for wireless-powered systems with massive MIMO," IEEE Transactions on Wireless Communications, vol. 17, no. 11, pp. 7159-7172, 2018.

[38] J. Fan and Y. Zhang, "Energy efficiency of massive MU-MIMO with limited antennas in downlink cellular networks," Digital Signal Processing, vol. 86, pp. 1-10, 2019.

[39] Y. Li, C. Tao, A. Mezghani, A. L. Swindlehurst, G. Seco-Granados, and L. Liu, "Optimal design of energy and spectral efficiency tradeoff in one-bit massive MIMO systems," arXiv preprint arXiv:1612.03271, 2016.

[40] Y. Huang, S. He, J. Wang, and J. Zhu, "Spectral and energy efficiency tradeoff for massive MIMO," IEEE Transactions on Vehicular Technology, vol. 67, no. 8, pp. 6991-7002, 2018.

[41] B. M. Lee, "Massive MIMO with Downlink Energy Efficiency Operation in Industrial Internet of Things," IEEE Transactions on Industrial Informatics, 2020.

[42] L. You, J. Xiong, X. Yi, J. Wang, W. Wang, and X. Gao, "Energy efficiency optimization for downlink massive MIMO with statistical CSIT," IEEE Transactions on Wireless Communications, vol. 19, no. 4, pp. 2684-2698, 2020.

[43] S.-N. Jin, D.-W. Yue, and H. H. Nguyen, "On the Energy Efficiency of Multi-Cell Massive MIMO With Beamforming Training," IEEE Access, vol. 8, pp. 80739-80754, 2020.

[44] A. a. R. T. Rajani, S, "Energy Efficient Data Detection For Uplink Multiuser Massive MIMO System," International Journal of Management, Technology And Engineering, vol. 8, no. 7, pp. 1169-1175, 2018.

[45] D. P. Bertsekas, "Nonlinear programming," Journal of the Operational Research Society, vol. 48, no. 3, pp. 334-334, 1997.

[46] D. P. Bertsekas and A. Scientific, Convex optimization algorithms. Athena Scientific Belmont, 2015.

[47] M. Gurbuzbalaban, A. E. Ozdaglar, P. A. Parrilo, and N. D. Vanli, "When cyclic coordinate descent outperforms randomized coordinate descent," 2017.

[48] O. El Ayach, S. Rajagopal, S. Abu-Surra, Z. Pi, and R. W. Heath, "Spatially sparse precoding in millimeter wave MIMO systems," IEEE transactions on wireless communications, vol. 13, no. 3, pp. 1499-1513, 2014.

[49] M. Cui, W. Zou, Y. Wang, and R. Zhang, "Low complexity joint hybrid precoding algorithm for millimeter wave MIMO systems," IEEE Access, vol. 6, pp. 56423-56432, 2018.

[50] Y. Won, J. Oh, J. Lee, and J. Kim, "A Study of an Iterative Channel Estimation Scheme of FS-FBMC System," Wireless Communications and Mobile Computing, vol. 2017, 2017.

[51] E. Björnson, E. G. Larsson, and M. Debbah, "Massive MIMO for maximal spectral efficiency: How many users and pilots should be allocated?," IEEE Transactions on Wireless Communications, vol. 15, no. 2, pp. 1293-1308, 2015.

[52] X. Meng, Y. Liu, X. Gao, and H. Zhang, "A new bio-inspired algorithm: chicken swarm optimization," in International conference in swarm intelligence, 2014: Springer, pp. 86-94.

[53] X. Liu et al., "Hybrid precoding for massive mmWave MIMO systems," IEEE Access, vol. 7, pp. 33577-33586, 2019.

[54] J. Du, W. Xu, H. Shen, X. Dong, and C. Zhao, "Quantized hybrid precoding for massive multiuser MIMO with insertion loss," in GLOBECOM 2017-2017 IEEE Global Communications Conference, 2017: IEEE, pp. 1-6.

[55] R. Pal, K. Srinivas, and A. K. Chaitanya, "A beam selection algorithm for millimeter-wave multi-user MIMO systems," IEEE Communications Letters, vol. 22, no. 4, pp. 852-855, 2018.

[56] S. Nagarani, J. Mala, and M. Kiruba, "An Enhanced Resource Allocation Scheme for MIMOOFDMA Wireless Network to Support Multiple Users with Reduced Interference Rate."

[57] X. Yu, Y. Du, X.-y. Dang, S.-H. Leung, and H. Wang, "Power Allocation Schemes for Uplink Massive MIMO System in the Presence of Imperfect CSI," IEEE Transactions on Signal Processing, vol. 68, pp. 5968-5982, 2020. 